\title{
Le droit penal de l'Union Européenne : discours inaugurateur
}

Citation for published version (APA):

Klip, A. H. (2004). Le droit penal de l'Union Européenne : discours inaugurateur. Maastricht University. https://doi.org/10.26481/spe.20041101ak

Document status and date:

Published: 01/11/2004

DOI:

10.26481/spe.20041101ak

Document Version:

Publisher's PDF, also known as Version of record

\section{Please check the document version of this publication:}

- A submitted manuscript is the version of the article upon submission and before peer-review. There can be important differences between the submitted version and the official published version of record.

People interested in the research are advised to contact the author for the final version of the publication, or visit the DOI to the publisher's website.

- The final author version and the galley proof are versions of the publication after peer review.

- The final published version features the final layout of the paper including the volume, issue and page numbers.

Link to publication

\footnotetext{
General rights rights.

- You may freely distribute the URL identifying the publication in the public portal. please follow below link for the End User Agreement:

www.umlib.nl/taverne-license

Take down policy

If you believe that this document breaches copyright please contact us at:

repository@maastrichtuniversity.nl

providing details and we will investigate your claim.
}

Copyright and moral rights for the publications made accessible in the public portal are retained by the authors and/or other copyright owners and it is a condition of accessing publications that users recognise and abide by the legal requirements associated with these

- Users may download and print one copy of any publication from the public portal for the purpose of private study or research.

- You may not further distribute the material or use it for any profit-making activity or commercial gain

If the publication is distributed under the terms of Article $25 \mathrm{fa}$ of the Dutch Copyright Act, indicated by the "Taverne" license above, 


\section{Discours inaugurateur}

\section{L e droit pénal de l'Union Européenne}

Versie 1 november 2004

\section{Introduction}

Le 7 février 1992, le Traité instituant l’Union Européenne fut conclut dans notre ville. Ce traité a induit une évolution par laquelle le droit pénal ne peut plus être considéré seulement d'un point de vue national. Consciemment ou non, les premiers pas vers un droit pénal communautaire ont été posés. Le sujet principal de mon exposé sera donc la signification de ce changement fondamental et structurel, d'un niveau national au niveau européen, pour les lois, la politique et la science du droit pénal. ${ }^{1}$

\section{La place du droit pénal dans la société}

Le droit pénal peut se féliciter d'une grande attention les derniers temps de la part des médias, du gouvernement et de la politique. Sur ce plan, le droit pénal se porte très bien. Les thèmes pénaux remplissent les nouvelles. Presque chaque citoyen a des opinions sur la façon dont un coupable devrait être puni. Je me réjouis du fait que mon domaine professionnel est un sujet tellement central dans la société. Mais toute cette attention a aussi ses inconvénients. Les résultats espérés sont souvent très élevés, trop même selon mon avis. ${ }^{2}$ Le droit pénal est souvent devenu, du moins dans les idéaux, le premier remède disponible contre les comportements indésirables. Cette évolution peut être observée non seulement aux Pays-Bas mais dans beaucoup d'autres pays, et certainement en Europe. ${ }^{3}$ J'ose même dire que l'union européenne est le protagoniste de l'idée selon laquelle le droit pénal est le premier remède contre la criminalité. L’union a exprimé cette pensée jusqu'à maintenant par un flot intarissable d'instruments pénaux.

Aux Pays-Bas, la proposition a été faite de sanctionner les parents pour une éducation négligente qui résulterait en un comportement criminel de la part des enfants. ${ }^{4} \mathrm{La}$ mutilation sexuelle des femmes á l'étranger sera bientôt sanctionnée, les femmes appartenant à des groupes à risque devront être contrôlées régulièrement. Aussi la prolongation de la prescription, renforce la toute puissance du droit pénal. Il s'élargit de jour en jour : par une augmentation des délits, des personnes intéressées, une place des délits et du temps. Le droit pénal a acquis comme instrument un niveau intouchable comprenant une dose substantielle de morale. ${ }^{5}$

Récemment des développements sont advenus, ceux la changent le statut de coupable, ils l'éternisent et le rendent absolu, par exemple; faire connaître au quartier le fait

\footnotetext{
${ }^{1}$ Ceci ne veut pas dire que l'Europe n'est elle-même point influencé par autres entités come les EtatsUnis. Voyez Joachim Vogel, Europäische Kriminalpolitik - europäische Strafrechtsdogmatik, Goltdammer's Archiv für Strafrecht 2002, p.517-534.

${ }^{2}$ P.J. van Koppen, Verankering van rechtspraak, Over de wisselwerking tussen burger, politie, justitie en rechter, discours inaugurateur Vrije Universiteit Amsterdam 2003, p.65.

${ }^{3}$ Voyez aussi J. de Hullu en E.A.M. Verheijen, De beoordeling van Eurostrafrecht, Delikt en Delinkwent 2002, p.696-697.

${ }^{4}$ Lettre du secrétaire d'Etat de la justice: forme d'aide pédagogique avec engagement, Première Chambre des Etats-Generaux 1999-2000, 27197, no.1, p.11.

${ }^{5}$ Buruma démontre la morale contenue dans des changements récents en matière de droit pénal. Y. Buruma, Grenzen aan de strafrechtelijke aansprakelijkheid, in: Glijdende schalen, Liber amicorum J.de Hullu, Wolf 2003, p.76.
} 
qu'un coupable d'attentat aux mœurs y réside. Un autre exemple, la possibilité d'annuler la nationalité néerlandaise des néerlandais de deuxième génération ou alors l'exclusion d'un coupable du marché du travail. Ces situations isolent des personnes pour toujours de la société. La science (et l'université) doit, par sa position dans la société, prendre parti contre ces évolutions et contre le fait que le droit pénal devrait se diriger vers ces solutions. Par contre, la science devrait expliquer au public que cela n’est pas la fonction du droit pénal et qu'une fois purgé sa peine, chaque coupable devrait pouvoir retourner inconditionnellement dans la société et que nous n'avons pas de place dans notre société pour une stigmatisation systématique ou pour des exils. ${ }^{6}$

Dans ces contextes il n'est pas étrange que la politique (européenne et néerlandaise) essaie de plus en plus de gagner du contrôle sur la pratique de sanction pénale. La politique essaye parfois de prouver que cette sanction n'est pas toujours dans de bonne main; des transactions concernant la fraude de certaines grandes entreprises devraient être selon le parlement sous contrôle du ministre de la justice. Avec l'établissement de peines fixes, le juge se verrait contraint à infliger des peines plus lourdes. ${ }^{7}$ Des hommes politiques, pouvant même être des membres du gouvernement, laissent parfois tomber des commentaires plutôt négatifs sur la façon dont certaines affaires individuelles ont été traitées. Cette critique ne vise pas le droit pénal luimême mais ses différents exécuteurs, la police qui n'attrape pas de criminels ; les procureurs qui ne poursuivent pas ou pour des délits trop bénins ou encore les juges qui infligeraient des peines trop clémentes. ${ }^{8}$

Pareil au niveau européen, la commission n'arrête pas de se plaindre des nonpoursuite de fraude : les instances nationales le font selon elle tellement mal qu'elle veut le faire elle-même. L'instauration d’une unité combattant la fraude était pour elle insuffisante, elle plaide depuis plusieurs années pour l'instauration d'un procureur européen. Ce sont toutes des façons d'agrandir le bras d'action du droit pénal. Mais elles méconnaissent la capacité du droit pénal en oubliant qu'il est impossible de poursuivre tous les délits. Il a été calculé qu'avec une capacité constante de l'appareil judiciaire, un pourcentage de plus en plus grand de crimes restera sans réaction. Boutellier parlait à ce sujet d'une sensation permanente de crise. ${ }^{9}$ De ce point de vue, la situation du droit pénal n’est pas si bonne.

\section{La tâche de la science pénale}

La science pénale doit se rendre compte de cette dernière constation, elle devra s'occuper de l'évolution et la formation du droit pénal. Ce que je dis ici n'est rien de nouveau, cela fait déjà des années qu’on en parle, par contre une nouveauté est le fait que l'influence de la science sur la législation et la politique diminue. Coment expliquer cela? Selon moi, cela est surtout dû à la différence dans la façon de de travailler de la science, d’un côté, et des médias prenant un rôle important dans notre

\footnotetext{
${ }^{6}$ Examinez aussi C.H. Brants, Over levende gedachten, discours inaugurateur 1999.

${ }^{7}$ Les mêmes juges doivent fonctionner en appliquant des normes de plus en plus vagues, ceci renforce le niveau d'indulgence qu'ils ont en prenant des décisions.

${ }^{8}$ Une enquête empirique intéressante sur la confiance des citoyens dans les décisions judiciaires: T. van der Meer, Vertrouwen in de rechtspraak, rechtstreeks 1/2004, p.9-55 et aussi P.J. van Koppen (p.1), qui fait remarqué que le droit pénal même est critisé, mais son élaboration de la argumentation montre que la critique se dirige aux fonctionnaires dans le reseau.

${ }^{9}$ H. Boutellier, De veiligheidsutopie, 2003, deuxième édition, p. 158.
} 
société, de l'autre. La façon rapide et "noire sur blanc" avec laquelle ces derniers cherchent une confrontation ne peut pas être facilement combiné avec les nuances et corrélations de notre métier.

La politique est dans un certain sens devenue dépendante des médias. Un homme politique proférant publiquement que tout le mal ne peut être battu sera presque défini par le publique comme quelqu'un considérant la criminalité comme sans importance. Lesquels aura l'audace de dire que la prospection d'un meurtre, vieux de 29 ans empêchera de poursuivre des crimes d'hier ou d'aujourd'hui ? $^{10}$

Les medias nous montrent souvent une image déformée de la réalité judiciaire. ${ }^{11}$ Cette image est même tellement divergente qu'elle mériterait une étude à elle seule. Je nomme par exemple la thèse non prouvée selon laquelle la réalité judiciaire aux PaysBas serait clémente. Par contre les faits pourraient nous prouver que notre règlement de libération anticipée est plus lourd que dans beaucoup d'autres pays européens, mais cela demandera une étude prolongée. Des journalistes continuent à écrire que des suspects sont libérés à cause des fautes de forme, mais ces dernières n'existent presque plus depuis les années quatre-vingt-dix. D’autres prétendent que des coupables sont libérés grâce à une bonne conduite, un critère inexistant aux Pays-Bas depuis vingt ans. L’exécution de la réclusion criminelle à perpétuité est à vie, néanmoins on lit que cette peine serait aux Pays-Bas pas pour toute une vie.

Les faits et la fiction s'entremêlent. La véritable situation n'est plus si importante, l'impression est ce qui compte. ${ }^{12}$ Dans la représentation de la criminalité, l'important est le fait qu'il sagit de criminels, il manque la notion que ce criminel européen est aussi un citoyen européen appartenant à la société européenne et ayant des droits de liberté. En faite, la stigmatisation revient du moyen âge dans notre société moderne. La considération des juges, dans leur décision pénale, de la façon dont le suspect a été traitée, est par ce fait justifiée. Les médias ne sont qu'une part de l'exécution de la peine et donc de la réalité. Je pense que cette évolution, dans laquelle la politique pénale est entre autres influencée par ce qui se passe dans les media, sera renforcée au niveau européen. La fiction pourra en devenir réalité. Ce trou béant peut mener à une surenchère d'espérance et de demande, suivie par de nouveaux pouvoirs, eux-mêmes suivis par une plus grande perte d'adhérence du droit pénal sur la société. Dans cette évolution sont aussi compris les dangers pour la sécurité juridique et la protection juridique. L'élargissement des pouvoirs ne renforce pas seulement la position de l'Etat mais aussi sa liberté de politique, tout cela agrandissant la possibilité de situations arbitraires. La tâche de la science consiste en démontrer ceci et rompre ce cercle vicieux. Cela est possible en contestant l'utilisation du droit pénal comme instrument de maintien de l'ordre.

\footnotetext{
${ }^{10}$ L'expérience en matière de poursuite de crimes de guerres nous montre que jusqu'à maintenant il y a eu de grands problèmes en matière de preuves en raison des grands espace de temps passé entre le crime et le jugement. Ceci a été résolu au tribunal de l'ex-Yougoslavie par des formes de participation plutôt facile voire: joint entreprise IK ZOEK DIT EVEN OP IN DE FRANSE VERSIE VAN HET ARREST. Dans lesquelles une espèce de raisonnement de culpa in causa est appliqué pour fonder la responsabilité criminelle. Pour des exemples ICTY, Judgement, Prosecutor v. Furundzija, Case No. IT-95-17/1-T, T. Ch.II, 10 December 1998, Klip/ Sluiter III-685, par. 216; ICTY, Judgement, Prosecutor v. Tadic, Case No. IT-94-1-A, A.Ch., 15 July 1999, Klip/ Sluiter III-761, par.220.

${ }^{11}$ Brants, p.34-36.

12 G.J.M. Corstens, Een stille revolutie, Arnhem 1995, p.14.
} 
Lorsque la criminalité est présentée comme un problème pouvant être résolu, nous risquons le fait que des moyens analogues à ce but irréalisable soient déployés. Je pense que le droit pénal devrait perdre sa forme intouchable et être réduit à un niveau beaucoup plus modeste. Ceci ne devrait pas nous mener jusq'au fait que des plaintes entendues dans notre société sur le défaut de sécurité seraient rangées comme naissant d'une "notion saine de droit de la part du peuple". Il y a encore un grand espace entre un viol fasciste du droit et le défaut total d'une notion ce dont la société aurait besoin. A la fin le droit pénal est là pour les besoins de la société et non pas seulement pour satisfaire certaines théories, il est non plus actif dans une situation stérile de laboratoire. Le droit pénal doit donc servir la société et pour cette raison il devrait être réel et convaincant. ${ }^{13}$ Dans ceci nous trouvons notre tâche sociale: démontrer l'acceptable et l'inacceptable. Continuer à pointer nos doigts sur les faits et les suites possibles, poser les bonnes questions, même lorsque l'explication coûte beaucoup de temps. Lorsque les hommes politique nous demandent des solutions introuvables, il faut leur répondre que la chose est impossible. ${ }^{14}$ La fosse entre science et politique ne m'inquiète pas, elle a toujours existé et ne fait autre que de démontrer la distance nécessaire. $^{15}$

Une autre facette du droit pénal pourrait aussi être critique. Dans un temps où les mœurs sont redessinées, le droit pénal pourrait aussi trouver un autre point de départ. Ceci pourrait au départ sembler menaçant, mais selon moi non moins important par le fait que nous avons perdu tout repère aux Pays-Bas. Beaucoup de notions qui autrefois étaient importantes, par exemple la distinction entre commettre un crime, être complice ou aider un criminel, ont l'air d'avoir perdu toute importance. Et je ne parle même pas encore de l'atteinte insuffisamment limitée de la "participation à une organisation criminelle », comme visée par l'article 140 du Code Pénal Néerlandais. Cette évolution est en contradiction avec les points de départ du droit pénal classique, qui lui exigeait un mouvement musculaire voulu, d'autre possibilité comme instrument de maintien de l'ordre n'étant acceptable que comme une dernière possibilité. En fait le principe que nul n'est responsable pénalement que de son propre fait est de plus en plus dépassé. ${ }^{16}$

Cela veut-il dire que le droit pénal actuel ne serait plus de notre temps ? Devons nous trouver de nouveaux principes ? Est ci cela est la conclusion à prendre, ne serait-il pas mieux d'en faire un point de vue européen ? Beaucoup de nouveautés technologiques sont nées depuis l'implantation de notre Code Pénal au dix-neuvième siècle. Les Pays-Bas ont une autre place géopolitique. Ceci avait été aussi remarqué récemment par notre ministre de la justice, il supposait que nous devrions passer à un système plus adapté à ce temps. ${ }^{17}$ Ceci me semble une bonne constatation. Mais la discussion fondamentale sur les points de départ manque encore. Pour moi personnellement le point de discussion suivant devrait être important: quel est le rôle que nous voulons donner au droit pénal dans notre société, comment voulons nous définir les notions de

\footnotetext{
${ }^{13}$ J. de Hullu, Recidive en straftoemeting, Deventer 2003, p.4.

${ }^{14}$ J. de Hullu, Recidive en straftoemeting, Deventer 2003, p.11.

${ }^{15}$ Examinez aussi Deuxième Chambre des Etats-Generaux, 1991-1992, 22268, no.5, p.5.

${ }^{16}$ Examinez D. Roef, Strafrechtelijke verantwoordelijkheid in de risicomaatschappij, in: P.L. Bal, E. Prakken, G.E. Smaers, Veiligheid of vergelding?, Deventer 2001, p.33-36.

${ }^{17}$ Le droit pénal dans un environnement changeant, collège du ministre de la justice Donner le premier décembre 2003 á l’Université Catholique de Nijmegen.
} 
culpabilité et complicité. Plus important que des détails comme la poursuite d'étrangers intégrés ici et étant des 'touristes sexuels', comme cela est rendu possible par l'article 5a du Code Pénal néerlandais. Dans une science abstraite comme le droit, rien n'est constant. Ceci peut nous mener à repenser des concepts pré-existants, mais avec la constatation que certains droits ne peuvent pas être rectifiés sans risquer de rendre certain principe de l'Etat de droit libéral inexistant.

\section{Quel est le rôle de l'Union Européenne dans le droit pénal ?}

Il est temps que je vous explique ce qui est pour moi le droit pénal européen, finalement c'est sur ce sujet que vous êtes venus m'écouter. Je voudrais définir le droit pénal européen comme l'ensemble de toutes les règles européennes ayant une dimension pénale. Nous pouvons distinguer trois niveaux différents:

1. un niveau national:

- l'application du droit communautaire (possibilité de choix: l'application par le droit pénal ou non, l'application elle-même est bien sûr obligatoire.), ${ }^{18}$ ainsi que

- la sanction de comportements illicites selon des règles déterminées par l'Union (comme formulées dans des actions communes, décisions cadres et conventions)

2. un niveau entre Etats:

- la régulation de la coopération administrative et judiciaire (laquelle mène en fait vers une application pénale communautaire)

3. un niveau supranational:

- le vrai droit pénal européen comprenant une application pénale directement européenne. Ceci est le cas concernant la concurrence illicite, mais des propositions ont été faites pour d'autres sujets. ${ }^{19}$

Dans la suite de ma dissertation je parlerais surtout du rapport entre le niveau national et supranational, ainsi que de la formation du droit pénal européen.

Il y a trente-cinq ans, pendant la veille de la complétion du réseau de convention concernant l'assistance juridique, avec lesquels les Etats se promettaient une aide mutuelle pendant chaque étape de la poursuite judiciaire, Van Agt supposait dans son discours inaugurateur un droit pénal extraverti 'que la sanction judiciaire d'Etats nationaux n'a pour but finalement non pas le maintien de société isolée, mais le bienêtre des personnes dans leur société s'étendant au-delà des frontières nationales. Charger le juge national de l'application des règles étrangères et conformes à cette opinion, pour autant que cela crée une administration de la justice plus juste et plus

\footnotetext{
${ }^{18}$ Examinez J.A.E. Vervaele, Handen en tanden van het gemeenschapsrecht, discours inaugurateur, Utrecht 1994.

${ }^{19}$ Je range selon l'interprétation de l'article 6 de La Convention européenne des Droits de l'Homme par la cour européenne des droits de l’homme, le droit de concurrence avec le droit pénal. Je suis donc un autre classement que Corstens, qui lui distingue le droit européen en trois componants: 1. la coopération pénale; 2. les droits de l’homme comme compris dans la convention européenne des droits de l'homme; 3. l'application du droit communautaire. Examinez G.J.M. Corstens, Europees strafrecht, in: Rede en Recht, Liber amicorum Nico Keijzer, Deventer 2000, p.11-25.
} 
efficace. ${ }^{20}$ Dix ans plus tard, en 1979, le même Van Agt faisait couler en tant que Premier Ministre néerlandais le plan du président français Giscard D’Estaing concernant un espace judiciaire $^{21}$. Beaucoup a changé depuis.

Premièrement, l'influence des conventions pénales a accru exponentiellement. J'ai recherché mathématiquement combien de temps les autorités néerlandaises avaient besoin pour conclure 50 traités relatés d'une façon ou d'une autre au droit pénal. Du 18 avril 1904 au 28 août 1954 (50 ans) ; jusqu'au 8 août 1975 (21 ans) ; jusqu'au 20 décembre 1988 (13 ans) ; jusqu'au 10 mars 1995 (presque 7 ans) ; jusqu’au 21 mai 1997 (2 ans) ; jusqu'au 25 mai 2000 (3 ans) ; jusqu'à septembre 2003 (3 ans et 4 mois). Il semble à première vue que la production internationale commence à devenir moins intense. Ceci n'est pas le cas. Ils naissent maintenant de plus en plus d'instruments de droit européen. J'ai débuté avec la tache énorme de recueillir et de catégoriser la masse d'actions communes, points de vue, recommandations, décisions et décisions cadres, mais sa complétion n'en est pas encore en vue. Pas seulement l'union européenne mais aussi d'autres organisations, comme les Nations-Unies, ont contribué à une situation ou les initiatives de la législation pénal sont plutôt pris au niveau supranational que national.

Je pense que ce changement continuera et surtout dans l'union européenne elle-même. La raison de ceci doit être recherchée dans le rôle social déjà nommée du droit pénal et le pied-d'estale sur lequel il est par les Etats membres : Selon l'article 29 du Traité sur l'union européenne: "l'objectif de l'union est d'offrir aux citoyens un niveau élevé de protection dans un espace de liberté, de sécurité et de justice... ». Cette ambition peut être vue comme la codification de l'utopie de sécurité si bien décrit par Boutelier. ${ }^{22}$ Elle accouple une émotion sociale avec une possibilité juridique et sera définie fortement la place du droit pénal dans la société européenne et le rapport entre le droit pénal national et européen. Le traité renforce aussi l'exigence de sécurité, très ressemblant à une exigence de consommateur, du citoyen directement envers l'union européenne. ${ }^{23}$ Autrement qu'aux temps de Van Agt et Giscard d'Estaing (du moins dans sa première vie politique), le droit pénal national se trouve sous pression par le fait qu'un moyen d'extermination de la criminalité semble introuvable. La souveraineté semble être vue comme un obstacle pour l'effectivité. ${ }^{24}$ Avec un peu de pessimisme, nous devrons nous attendre au fait que la politique ne pourra pas offrir de grande résistance contre la pression croissante de passer au droit pénal européen. Ensuite cela fera croître la pression sur l’Union Européenne de prendre de plus en

\footnotetext{
20 A.A.M. van Agt, Naar een extravert strafrecht, discours inaugurateur, Katholieke Universiteit Nijmegen 1969.

${ }^{21}$ Examinez Annexe Deuxième Chambre des Etats-Generaux, 1978-1979, no.1261. A. Weyemberg, L'harmonisation des législations: condition de l'espace pénal européen et révélateur de ses tensions, Bruxelles 2004, p.14-15.

${ }^{22}$ Examinez aussi H. Boutellier, De veiligheidsutopie, deuxième édition 2003, p.47. Cette espace est aussi stipulée dans les articles 41 et III-158 du project de la Constitution pour l’Europe.

${ }^{23}$ Ceci nous amène à la question suivante: reste-il de la place pour que le citoyen européen créer luimême de la liberté, sécurité et justice ? Ici aussi un changement, dans le traité d’Amsterdam, l’Europe devait se rapprocher du citoyen et dans le projet de constitution européenne le citoyen doit être amener plus près de l'Europe.

${ }^{24}$ Examinez par exemple C. Rijken, Trafficking in Persons, dissertation Universiteit van Tilburg 2003, p.9.
} 
plus en main propre. Du traité de Maastricht se déduit un intérêt plus grand du droit pénal dans notre société, et surtout de la version européenne. ${ }^{25}$

Le droit pénal est-il entre de bonnes mains sous contrôle de l'union européen ? La réponse là-dessus est pour le moment, négative. Il existe en effet beaucoup de loi au niveau de l'Union existant apparemment sans raison. En 1997, peu de temps après l'arrestation de Dutroux, une action conjointe a été déclaré qui aurait pour but le combat de réseaux internationaux de pédophilie. Pourtant, le procès actuel ne comporte aucun aspect international. ${ }^{26}$ Naissant comme réaction à l'attentat du 11 septembre, la mesure proposée, le mandat d'arrêt européen, serait la solution au fait présume que trop d'extraditions seraient refusées entre autres parce que l'interdiction de double peine formerait un obstacle. Jamais des données ont put prouver cette supposition. Il existe donc de la législation contre des problèmes inexistants. ${ }^{27}$ Les recherches comparatives, que l'union effectue pour supporter un projet de loi ou pour la facilitation d'implémentation de directives, ne sont pas suffisantes. Elles ne dépassent jamais le niveau de comparatisme normatif et laissent de côté l'aspect réel de l'application. ${ }^{28}$ Le phénomène du tableau de score, là où les Etats doivent rapporter leurs progrès d'approbation et implémentation de règles et les institutions charger de l'application de ces règles, fait beaucoup penser au système de planifications de l'ancienne Union soviétique. Là aussi formellement tous les buts étaient atteints et la réalité cachée. L'accroissement d'importance de UE dans le droit pénal devrait selon moi être en rapport avec une vraie compréhension de la criminalité européen. Fijnaut a déjà proféré qu'il trouve un territoire de recherche européen important. ${ }^{29}$ Pour autant qu'il veuille dire par la plus de recherche comparatisme et criminologue en Europe et le contrôle et soutien de politique, je suis tout à fait d'accord avec lui. ${ }^{30}$ Le manque d'un Centre de recherche scientifique et de documentation européenne est très ressentie dans ce domaine. ${ }^{31}$

L'union normalise l'application des lois, mais pas d'un point de vue critique envers le pouvoir, seulement d'un point de vue répressif. Ceci mène a une malformation d'autant pour l'application que pour la sûreté juridique. Je trouve cela un grand problème : pas l'origine européenne forme un souci mais le manque de qualité et la quantité de lois pénales Européennes m’alarme. La machine législative de l’Union

\footnotetext{
${ }^{25}$ La liberté Européenne nourrit l'insécurité, voyez: Ralf Dahrendorf, De Volkskrant 16 april 2004, p.13.

${ }^{26}$ Examinez A. Weyembergh, p. 344: “les grandes difficultés auxquelles sont confrontés les travaux de rapprochement des législations pénales accroissent la tentation de suivre l'événementiel et d'exploiter les drames de l'actualité pour obtenir le consensus nécessaire, ce qui favorise le recours à des définitions larges négociées et adoptées dans l’urgence, sans réflexion approfondie.”

${ }^{27}$ A.H.J. Swart, Een ware Europese rechtsruimte, Discours inaugurateur Amsterdam 2001, p.29. Swart se demande aussi si il y a une relation avec la bonne volonté des Etats membres de faire de cette coopération un succès.

${ }^{28}$ Sur la méthode de recherche comparative en droit pénal: C.J.C.F. Fijnaut, Rechtsvergelijking en strafrecht(swetenschap): enkele methodologische beschouwingen, Preadvies voor de Nederlandse vereniging van rechtsvergelijking, no.61, Kluwer 2001.

${ }^{29}$ Examinez Fijnaut, preadvies, p.88.

${ }^{30}$ Examinez C. Fijnaut, De Europese Unie: een lusthof voor (strafrechtelijke) rechtsvergelijking, Deventer 2001; idem De uitbreiding van de Europese Unie en de strafrechtsvergelijking, in: De meerwaarde van de rechtsvergelijking: Opstellen aangeboden aan prof. mr. H.U. Jessurun d’Oliveira, p.201-213.

${ }^{31}$ Par contre il existe un centre européen observant les problèmes de drogue et un autre pour le racisme et la xenofobie.
} 
rends le droit pénal européen de plus en plus compliqué et inaccessible. L’Etat de droit européen s'avérerait le mieux servi par une pause législative. Déjà, souvent j’ai dénoncé, sans aucun succès d'ailleurs, le fait qu'aucune réflexion ne prenne place, jamais les effets possibles d'un certain instrument ne sont attendus. ${ }^{32}$ Comme exemple récent je nomme la proposition décision-cadre relative au mandat européen d'obtention de preuves tendant à recueillir des objets, des documents et des données en vue de leur utilisation dans le cadre de procédures pénales? Dans ce papier des traités datant de 2000 et 2001 sont déja remplacés avant leur implémentation. Ainsi que la décision Dutroux, qui elle aussi a été remplacée par une décision cadre de décembre 2003. ${ }^{33}$

Les activités de l’Union Européenne sur le terrain pénal présentent une tendance à se diriger d'un problème a une solution, mais aucun système ou cohérence ne peut y être retrouvé. Opérer vite et symboliquement est estimer mieux qu'agir raisonnablement et effectivement. Je trouve donc aussi que le droit pénal européen n’a, jusqu'à ce jour, a fonctionner á contre-sens au détriment du maintien d'ordre et de la protection des droits du citoyen européen. Un collègue allemand déterminer ceci comme le droit pénal Systemschutz : «Á l’horizon on voit un droit pénal Européen symbolique et une justice Européenne symbolique. Expressant l'incompétence de la politique, qui n’a pas assez de force pour une reforme structurelle de la societé. ${ }^{34}$ Il voulait dire que l'utilisation du droit pénal pour des fins politiques maintien l'ordre politique mais ne mène pas à une utilisation réelle du droit pénal. J'ajoute que cela implique le danger que l'ordre de papier conçu de cette manière ne soit pas utilisable dans la pratique ce qui mènera à une application plutôt arbitraire. Ceci ne peut autre que faire honte a la fonction protectrice de l'Etat de droit.

\section{Que faire maintenant ? Que pourrait appartenir au droit pénal européen ?}

Pour l'occasion de l'ouverture d'Eurojust, aujourd'hui il y a un ans précisément, le Ministre de justice néerlandais a proposé de créer une compétence européenne spéciale pour certain cas dépassant les frontières et laissait le reste aux juridictions nationales. $^{35}$

Son opinion peut être ramenée à ceci : c'est inutile de vouloir poursuivre toute la criminalité au niveau européen car 90 pour-cent n’a de conséquences qu'á l'intérieure des frontières. ${ }^{36}$ Sa proposition n'étant pas encore très détaillée, elle en reste au moins très intéressante. Certainement maintenant, au milieu de la discussion sur la constitution européenne et de la prochaine présidence néerlandaise, pendant laquelle d'autres initiatives peuvent être attendues.

\footnotetext{
32 A.H. Klip, Amsterdams uniestrafrecht, Nederlands Juristenblad 1998, p.811-816; Neuere Entwicklungen im europäischen strafrecht und in der europäischen Zusammenarbeit in Strafsachen, in: 22. Strafverteidigertag vom 20.-22. März in Erfurt, Schriftenreihe der Strafverteidigervereinigungen, Köln 1999, p.39-54. Voyez aussi C.J.C.F. Fijnaut, De strafrechtelijke bescherming van de financiële belangen van de Gemeenschap tegen fraude, Delikt en Delinkwent 2000, p.972-988.

${ }^{33}$ Decision-cadre du 22 décembre 2003 relative à la lutte contre l'exploitation sexuelle des enfants et la pédopornographique, JO 2004, L 13/44.

${ }^{34}$ P.-A. Albrecht, Europäischer Strafrechtsraum: Ein Albtraum?, Zeitschrift für Rechtspolitik 2004, p.1-4.

${ }^{35}$ Quelque jours plus tard ce point de vue a été répeté dans la note : “Un espace judiciaire Européen” du 9 mai 2003 de la part du secrétaire d’Etat des affaires Européenne, kenmerk DIE-258/03.

${ }^{36}$ Examinez aussi Ch.J. Enschedé, A Model Penal Code ?, Report for the Council of Europe 1971, reprinted Arnhem 1990.
} 
Une distinction entre un droit pénal européen et national suppose selon moi un système pénal européen complet, donc avec un juge pénal européen, un ministère public européen et une police européenne, tout ça fondé sur la législation européenne et vérifié par un parlement européen librement élu. Si nous entreprenons ceci, cela exige une conceptualisation de l'européen et du national ou de ce qui devrait l'être. ${ }^{37}$ C'est une chose nouvelle, que les Pays-Bas, en tant que président de l'union européenne, pourraient introduire au débat. Mais la pétition du ministre de la justice sortie le mois dernier ne continue pas sur le même chemin que l'idée de délimitation prononce. ${ }^{38}$ Le gouvernement garde la condition de la double incrimination pour la coopération avec d'autres Etats membres lorsque le fait a été commis aux Pays-Bas, ce qui veut dire que si le fait n'est pas incriminé ici, les Pays-Bas ne peuvent pas fournir d'aide judiciaire.

Je voudrais proposer un autre model, celui-là pourrait avoir cette allure : Si le problème est le fait que l'étranger ne devrait pas pouvoir exiger juridiction sur un fait commis ici mais pas incriminé ici, alors la limitation de la juridiction devrait être le principe a être suivi. Cela mène à une toute autre règle : la juridiction n'a besoin être établie sur son propre territoire. ${ }^{39}$ L'Allemagne ne peut à ce moment plus poursuivre un de ces citoyens utilisant de la drogue aux Pays-Bas. Mais cela veut aussi dire que si les Grecs trouvent utile d'incriminé le fait de marquer des numéros de registration des avions, nous nous trouvons obligé de les aider avec l'application de cette règle. ${ }^{40}$ Cela demande beaucoup de confiance mais a de grands avantages envers le maintien de l'ordre et la sûreté juridique. Il est alors toujours claire quel Etat est élu pour l'application de la loi. La situation actuelle de juridiction recouvrante mène à un débat constant entre Etats membres a propos de la pénalité de certains faits et sur la politique pénale à manier. ${ }^{41} \mathrm{Si}$ nous supprimons la double pénalité, et limitons la juridiction au territoire national, nous pouvons en plus mieux continuer à bâtir un réseau international de coopération pénal. ${ }^{42}$ Le système du conseil d’Europe n'a en effet jamais réussi a incorporer un certain thème : Un règlement pour les conflits de juridiction. ${ }^{43}$ L'accroissement des juridictions au-delà des frontières nationales a été conçu pour empêcher les suspects d'éviter toute poursuite et pour que chaque suspect doive rendre ses comptes dans son propre pays, par pour changer la politique

\footnotetext{
${ }^{37}$ Je souligne le point de vue du gouvernement Néerlandais qu'une distinction claire est favorable. Deuxième Chambre des Etats-Generaux, 2003-2004, 28473, no.35, p.4.

${ }^{38}$ Notitie: Het vereiste van dubbele strafbaarheid in het Nederlandse strafrecht, Deuxième Chambre des Etats-Generaux,, 2003-2004, 29451, no.1; Proposition décision-cadre relative au mandat européen d'obtention de preuves tendant à recueillir des objets, des documents et des données en vue de leur utilisation dans le cadre de procédures pénales, 25 novembre 2003, 15221/03.

${ }^{39}$ Une pareille nécessité pourrait exister pour l'enquête judiciaire par delà de frontières.

${ }^{40}$ Une approche pareille est aussi plus conforme au principe de légalité.

41 Alors les Pays-Bas pourront aussi s'éloigner d'une politique d'attente et de distance envers des initiatives pénales européennes. Par exemple le gouvernement néerlandais dans sa note droit pénal et procédure pénale en Europe, Deuxième Chambre des Etats-Generaux, 1998-1999, 26656, no.1. Voyez aussi P.H.P.H.M.L. van Kempen, Waarborgen tegen de onwrichtende werking van Eurostrafrecht, Glijdende schalen, de Hullubundel, p.247-266.

${ }^{42}$ A.H.J. Swart, Goede rechtsbedeling en internationale rechtshulp in strafzaken, discours inaugurateur, Deventer 1983.

${ }^{43}$ Des essais de systématisation du système par un rangement de principe juridictionel ont ratés, mais nous apprennent beaucoup. Voyez Conseil de l'Europe, Juridiction extraterritorial. Avec cette méthode l'Union Européenne pourrait preventir conflits de juridiction, comme l'article 31 sub c de la Traité de l' Union Européenne demande.
} 
pénal d'un Etat. Finalement peu de délits sont considérés si différemment, la plupart des délits sont incriminés d'une façon au d'une autre partout en Europe.

La ou il reste des différences, cela a certainement des raisons. La délimitation proposée à l'instant par moi ne concerne que les rapports entre différents Etats membres. Elle ne dit rien encore sur les cas difficiles dont les locus délicti ne peuvent par être situé uniquement dans un Etat. ${ }^{44}$ Ces cas la devrait être réglée au niveau européen. Nous devrons donc formuler un critère pour la réglementation au niveau européen.

Dans sa note «un espace criminel européen » de l'année dernière, le gouvernement proposait ceci : «une lutte effective de la criminalité envers les intérêts européens (falsification de l'euro et détournement de fonds contre les intérêts financiers de la communauté) et des crimes graves dépassant les frontières, ceux-ci devraient être spécifiés mais pourrons varier de trafique de bien (il)licite et de crimes contre l'environnement, aura finalement besoin d'une juridiction propre et homologue qui ne sera pas limitée par les frontières sociales. » ${ }^{45}$

En fait le ministre de la justice demande un point de vue criminologique en rapport avec les formes par lesquelles la criminalité apparaît et surtout en rapport avec la question si cette criminalité dépasse oui ou non les frontières. ${ }^{46}$ Cela me semble la bonne voix. Je trouve la méthode présente, premièrement créer une organisation: Europol, le ministère public européen ou alors introduire des incriminations dérivés de problèmes étant à la une des médias avant un top européen, une méthode pas vraiment réfléchie. Car elle n’influence que la façon dont le public voit le droit pénal, pas le droit lui-même.

La démarcation doit être recherchée dans le droit matériel, pas dans les conditions de coopération. Pour cette raison les délits qui feront partie d'un droit européen pénal devront répondre à certaines conditions. Lesquels ? A mon avis il s'agit de ces trois conditions cumulatives :

1. Les délits ont selon sa manifestation un caractère dépassant les frontières nationales;

2. La prévention, dépistage et jugement d'un de ces délits rencontrent au niveau national plus de difficultés qu'au niveau européen;

3. Les délits ont un rapport avec le secteur d'intervention européen. ${ }^{47}$

\footnotetext{
44 Sans parler du problème des différentes doctrine de locus delicti. Examinez H.D. Wolswijk, Locus delicti en rechtsmacht, diss. Utrecht 1998.

${ }^{45}$ Note de 9 mai 2003, voyez note 35 supra.

${ }^{46}$ Examinez aussi D. van Eck, Het universele karakter van het strafrecht, opstellen over recht en rechtsgeschiedenis, aangeboden aan D. Hermesdorf, Kluwer 1965, p.149-150: "l'évolution vers un droit pénal international, si cela veut dire que la concrétisation des normes ou leurs application quittera partialement le niveau national, devra être un changement, décidé par le critère du système pénal opérant le plus responsablement envers la société. Mais en prenant cette décision, il ne faudra pas oublier que l'application du droit pénal n’ait que une des maintes taches dédier à la société. La décision sur le partage des taches ne devra donc être prise purement à partir de critère d'effectivité d'application pénal. Cette devra être prise à partir d'un contexte plus large, comprenant aussi les autres tache que la société a prise sur elle”.

${ }^{47}$ Examinez aussi mon texte: Conditions for a European Corpus Juris Criminalis, in Michael Faure, Jan Smits and Hildegard Schneider (eds.) Towards a European Ius Commune in Legal Education and Research, Intersentia 2002, p.111. La rédaction des ores est souvent de l’origine communautaire.
} 
Ici sont concernés les faits avec un locus delicti dans plus d'un Etat, donc aucun de ces Etats n'est plus apte à prendre des mesures. Entre autres je pense au droit pénal douanier, le détournement de subventions, le blanchissement d'argent, le trafic de femmes et hommes et le trafic de drogue et d'armements. La deuxième condition concerne l'efficacité. Si le délit est un cas dépassant certaines frontières nationales sans que cela pose aucun problèmes aux autorités nationales pour la poursuite judiciaire, je ne vois aucune raison pour laquelle le cas serait réglé au niveau européen. ${ }^{48}$ Le troisième élément sers à souligner le fait que les différentes formes de maintien doivent être vues en cohésion, mais souligne aussi le principe de la subsidiarité.

Bien sur, il y aura des suspects qui ne feront pas attention a cette distinction minutieuse en préparant et commettant un crime. Ils surgiront des ensembles de faits comprenant un aspect européen et national. Le principe d'une bonne administration de justice, qui a été inspiré par la transmission des poursuites judiciaires, pourra aussi être déterminant pour la recherche d'un forum de jugement convenant. ${ }^{49}$

Donc l'espace judiciaire européen mène à trois sphères de droit bien délimité: une nationale, une européenne et une extra-européenne. ${ }^{50}$ Les plans de la commission européenne pour un ministère public européen comme décrit dans le corpus iuris m'enthousiasme donc moins. Cela met en place un système hybride, entre une application national et européenne. ${ }^{51}$ Les articles III-171 et suivant du projet de constitution européenne ne proposent aucun nouveau regard là dessus et renforce surtout cet aspect hybride. Des réservations ressemblantes existe envers la solution américaine ou la délimitation reste vague, ce qui emporte beaucoup de malentendus juridictionnels. $^{52}$ Si nous décidons d'implanter un nouveau droit pénal européen l'influence européenne sur le droit pénal national est beaucoup plus limité. ${ }^{53} \mathrm{Et}$ l'objection envers la possibilité de non-respect des priorités nationales de la part des autorités européenne plus puissantes comme Europol ou Eurojust disparaît. ${ }^{54}$

Mais tout n'est pas en rose vue d'un tel point de vue. Un système européen propre a aussi des inconvénients. De nouvelles institutions sont crées, ce qu'il fait accroître le flot d’informations. Aussi, un système pareil doit être accepter par les autorités

Voyez J.A.E. Vervaele, Handen en tanden van het gemeenschapsrecht, discours inaugurateur Utrecht 1994, p.36.

${ }^{48}$ Examinez Première Chambre des Etats-Generaux, 2003-2004, 28350 (R1720) et 28351, B, p.2.

${ }^{49}$ A.H.J. Swart, Goede rechtsbedeling en internationale rechtshulp in strafzaken, discours inaugurateur Deventer 1983.

${ }^{50}$ Examinez aussi, le ministre de la justice, Première Chambre des Etats-Generaux, 2003-2004, 28350 (R1720) et 28315, B, p.4.

${ }^{51}$ Ceci a été nommé comme ceci par le gouvernement grâce à une grande pression du ministère publique. Examinez Première Chambre des Etats-Generaux, 2003-2004, 28350 (R1720) et 28351, B, p.2.

52 Déja en 1971 Enschedé rejetait le American Model Penal Code comme model pour un European Model Penal Code, examinez Arnhem 1990.

${ }^{53}$ Dans différentes notes sur le droit pénal européen, le gouvernement a donné un compte rendu de principes néerlandais que le gouvernement trouve intouchable. Voyez Notitie betreffende de verhouding gemeenschapsrecht - strafrecht, Deuxième Chambre des Etats-Generaux, 1991-1992, 22300 VI, no.39; Notitie Strafrecht en strafprocesrecht in Europees verband, Deuxième Chambre des Etats-Generaux, 1998-1999, 26656, no.1.

${ }^{54}$ Le ministre de la justice en a parlé déja dans son discours à Eurojust, le 29 avril 2003. 
nationales. ${ }^{55}$ Une nouvelle institution ne doit pas être vue comme menaçante ou concurrente ce qui la laissera dépourvu d'information. La position d'Europol me laisse de ce point de vue plutôt pessimiste.

\section{Que signifie la situation actuelle pour l'harmonisation et la reconnaissance mutuelle?}

Pour le model choisi par moi, un model comprenant un droit pénal national et un autre européen, ayant chacun une juridiction sur différents délits, l’harmonisation de droits pénaux européens n'est pas indispensable. L’harmonisation actuelle du droit national a déjà souvent été critiquée, est cela de bon droit. Elle a pour fondation opaque l’idée que la double incrimination est un obstacle pour la coopération pénal. La méthode choisie à un effet inversé. La vraie influence du model d'harmonisation reste pour cette raison moindre, si non absent. ${ }^{56}$ Cela a tout d'abord un rapport avec le caractère des lois issues de compromis. Les instruments sont souvent vague et non obligatoire. Chaque Etat membre garde une grande liberté d'interprétation. L'implémentation est une autre raison de la différence de maintien entre Etats. Un Etat qui n'aurait pas réussi à gagner une certaine négociation peut toujours implanter selon une façon très «national » et par la suivre son point de vue. ${ }^{57}$ La troisième raison est l'absence d'un juge commun. Le rôle définitif de la cour de justice européenne dans l'interprétation des décisions cadre doit encore être définie. La quatrième raison est l'utilisation des langues authentique. Ceci mène finalement à une grande différence entre les formes de maintiens nationales.

Apres demain l'union européenne s’agrandira de 15 a 25 membres, de 375 millions d'habitants à 450 millions d'habitants. Cette taille ne facilite pas la tâche de trouver un compromis. La force unissante de l'harmonisation décroisera encore plus.

Partons de sphères juridiques distinctes: Une européenne pour les délits spécifiquement européens et diverse juridiction national pour les délits nationaux, dans ce cas là et avec l'absence de juridiction extra-territorial, les conditions de double incrimination ne sont plus nécessaire. Avec ceci expire la raison de l'harmonisation du droit pénal matériel. Selon mes prédictions l'harmonisation de certains aspects du droit de procédure pourrait être constructive. Ceci exige quelques explications.

Si les Etats membres acceptent les un des autres que la pratique des juridictions est exclusive, cela pourrait mener plus facilement à une reconnaissance mutuelle inconditionnelle d'ordres et d'extraditions de personnes et preuves ou d'autres décisions. Dans un système de reconnaissance mutuelle les murs de distinction entre systèmes pénaux tomberont. ${ }^{58}$ Pour en arriver à une pratique entièrement libre quelques réservations devront disparaître. Ainsi les conditions pour l'interpellation de suspect différent d'Etat en Etat. Ceci met de la pression sur un Etat qui pose des

\footnotetext{
55 Examinez: A.H. Klip, Geïntegreerde bescherming van de financiële belangen van de EG; verschuiving van de staat-staat-benadering naar verticalisering van de procedure?, in: J.A.E. Vervaele (ed.), Transnationale handhaving van de financiële belangen van de Europese Unie, Intersentia 1999, p.99-111.

${ }^{56}$ Examinez par exemple, T. VanderBeken, From Brussels with Love, Bespiegelingen over de invloed van de Europese Unie op het Belgisch strafrecht, Preadvies voor de Vereniging voor de vergelijkende studie van het recht van België en Nederland 2002. Voyez Weyembergh, p.336.

${ }^{57}$ Examinez Weyembergh, p. 342.

${ }^{58}$ A.H.J. Swart, Een ware Europese rechtsruimte, discours inaugurateur Deventer 2001, p.24.
} 
conditions plus lourdes. Des problèmes analogues surviendront dans la pratique entre instruments de forces et l'acquisition de preuves. Le rapprochement des conditions pour la fouille ou la confiscation enlèvera les réserves sur la reconnaissance mutuelle d'un ordre étranger.

Des constatations comme celles ci prouvent la nécessité d’une conceptualisation du principe de reconnaissance mutuelle. Mais qu'est ce qui doit être reconnu? Quelles conditions matérielles pour l'application de moyen de force ont été respectées? Qu'une certaine autorité a constaté ceci? Qu'un Etat se plie aux demandes de procédures pour l'acquisition de preuves? Et pourquoi l'exécution obligatoire d'une demande étrangère devrait être prioritaire envers le propre système judiciaire ? Bref, la notion doit être conceptualisée.

Dans la situation actuelle l'Etat prenant l'initiative oblige l'autre Etat de reconnaître quelque chose. Pourquoi dans un système de reconnaissance mutuelle, l'Etat poursuivant devrait avoir une priorité au-dessus des Etats ayant décidé de ne pas poursuivre ? Cela disparaîtra avec la limitation des juridictions.

A quoi devrait ressembler le droit pénal européen ?

Ci-dessus j’ai parlé de la distinction entre le droit pénal européen et national, mais je n'ai encore rien dit sur la forme que pourrait prendre ce premier. A quoi pourrait-il ressembler?

En faite deux méthodes sont évidentes pour la stipulation de la forme et substance que devrait prendre le droit pénal européen matériel et la procédure pénal. A ce niveau nous devons aussi penser à la procédure car finalement nous parlons de la création d'un nouveau système. La première méthode consiste à observer la création pénale de l'union européenne. La deuxième consiste à observer les différentes traditions pénales des Etats membres. Elles seront toutes les deux tout sauf simples. Cela n'a rien de surprenant. Ce sera aussi la troisième fois depuis notre calendrier que nous essayons cela en Europe. La situation a beaucoup changer depuis le droit pénal roman canonique et le Code Pénal de Napoléon.

Que peut-on observer dans la production d'instrument pénal européenne jusqu'à ce jour? La part générale en est une qui peut être vue comme droit pénal de responsabilité de son propre fait. ${ }^{59}$ Le vrai comportement du coupable n'est plus si importante, plus l'est la position et la responsabilité prise sur soi même (violation de diligence). Le droit d'organisation économique comme fondement y joue probablement un rôle. Les instruments européens ont des éléments que l'on retrouve dans tout les droit national dans leur part générale. A cause du manque de réflexion là-dessus au niveau européen, beaucoup de petits bouts indépendants existent. Ils disent quelque chose sur la façon dont l'union définie «commettre un crime » et la façon par laquelle les crimes devraient être décrits. Presque sans exception cela comprend des formes de participations largement définies. La condition de l'intention est objectivée par l'apparence du comportement. (Art.1 point 4 de la Convention relative à la protection des intérêts financiers des Communautés européennes). Et aussi la responsabilité de personne morale et de leurs direction, formelle et de facto, devrait être établi (Art. 6 de la décision-cadre relative à la lutte contre l'exploitation

\footnotetext{
${ }^{59}$ Une évolution observée très fortement dans les tribunaux pénaux internationaux.
} 
sexuelle des enfants et la pédopornographie). Si le pouvoir de représenté un personne morale, de prendre des décisions en son nom ou d'exercer contrôle sur elle et pénalisé, cela diminuera la significance de l'irresponsabilité pénale. Dans l'appréciation de l'imputabilité á la personne du delinquant de son acte comprend aussi un avis sur la possibilité d'irresponsabilité pénale. On peut aussi remarquer, surtout dans les préambules, certains éléments de politique pénal, dont l'influence est ressenti dans le droit pénal substantiel : L’Union Européenne s'efforce d'aboutir á l'élimination du comportement criminelle(ou peut être même des criminelles) et la prévention de récidives.

Cette méthode nous offre que peut de perspicacité pour le système de procédure pénal a développer. L'acquis du troisième pilier ne comprend encore que peut de disposition de nature procédurale. Ceci est compréhensible car l'application des lois pénale se passe encore strictement au niveau nationale. Ici, l'accent est surtout mis sur l'harmonisation et facilitation de la coopération.

La première méthode pour designer ce qui devrait appartenir au droit pénal Européen consiste à déduire certaine chose du droit Européen préexistant. La deuxième consiste à distiller un nouveau droit Européen à partir des différentes traditions pénale des Etats membres. ${ }^{60}$ Ici aussi la part générale du droit pénale (donc la personne du deliquant et la complicité, la délibération et la faute etc.) et la procédure pénal sont plus importants que les pénalisations spécifiques. ${ }^{61}$ L'article 6 du traitée de L’EU nous offre une base pour une déduction á partir des traditions pénale des Etats membres. Sinon une jurisprudence de la Cour de Justice des communautés Européennes sur le sujet des traditions constitutionnelles et pénales des Etats membres c'est formée ensemble avec des principes généraux du droit communautaires. ${ }^{62}$ Que nous offre une première inventorisation des traitions pénales? Premièrement elle nous montre les droits fondamentaux importants pour le droit pénale. Ici, grâce au rôle harmonisant de la CEDH, une certaine conformité existe. Par contre si nous examinons d'autres aspects du droit pénale, cette conformité n’est pas retrouvée. $^{63}$

De grandes différences culturelles existent malheureusement, ce qui rend la recherche du commun difficile. De règles plus au moins ressemblantes peuvent interprétées de façon très différentes. Par exemple le principe du légalisme, la règle voulant dire que nulle crime peuvent être punis de peines qui n'étaient pas prononcées par la loi avant qu'ils ne fussent commis, mènera á de différentes interprétations des normes sur l'élaboration de textes claires et précis (Lex certa). La culture juridique est aussi la façon dont on perçoit le droit étranger. Par exemple, les pays ayant un principe

\footnotetext{
${ }^{60}$ Une méthode de déduction comme celle ci et comparable avec l'harmonisation. On pourrait dire que la déduction à partir de différents principes nationaux a un certain effet d'entonnoir. Dans la méthode d'harmonisation classique par contre, l'entonnoir marche en sens inverse.

${ }^{61}$ W. Bottke, Die Struktur von Täterschaft bei aktiver Begehung und Unterlassung als Baustein eines gemeineuropäischen Strafrechtssystems, in: Bausteine des europäischen Strafrechts, CoimbraSymposium für Claus Roxin, 1995, p.235-246.

${ }^{62}$ Par exemple: CJCE, 5 mars 1980, Ferwerda, C-256/78, Jur.1980, 623: CJCE, 13 novembre 1990, Fedesa, C-331/88, Jur.I-4057; CJCE, 4 octobre 1991, SPUC, C-159/90, Jur.1991, 4658; CJCE, 14 mai 1974, Nold, C-4/73, Jur.1974, 491; CJCE, 13 decembre 1979, Hauer, 44/79, Jur.1979, 3727; CJCE, 21 septembre 1989, Hoechst, C-46/87 et C-227/88, Jur.1989, 2859.

${ }^{63}$ Il y a bien plus de droit de procédure pénal que celui comprit dans la convention européenne des droits de l’homme. Examinez M. Delmas-Marty, Procédures pénales d’Europe, Paris 1995.
} 
d'opportunité critiquent les pays ayant un principe de légalité en disant que ces derniers mèneraient vers une rigidité et vers une application démesurée du droit, dans l'autre sens la critique est que l'opportunité peut mener à l'arbitraire. En réalité les deux systèmes ont changé subtilement leur système pour diminuer ces critiques. Le débat récent aux Pays-Bas sur les peines fixes est aussi un bon exemple sur la perception du droit étranger. Ce débat a été guidé par la vision néerlandaise d'une pratique d'exécution de peines rigides (toujours $2 / 3$ des peines exécutées). Les pays connaissant des peines minimales ont par ailleurs beaucoup d'exceptions sur ce minimum. La France a aboli les peines fixes car elles obligent les juges à trouver le suspect coupable de crimes moins graves lorsqu'ils trouvent la peine fixes du crime «original » trop lourde. ${ }^{64}$

Ceci ne sont que des exemples mais ils nous montrent qu'une quête envers le plus grand commun diviseur nous mènera vers un système entièrement nouveau, là où aucun système national ne sera entièrement reconnaissable. Concernant la méthode de développement d'une nouvelle procédure pénale, les expériences de divers cours pénaux internationaux peuvent ce révéler très éducatives.

Il existe un autre aspect de la création d'un droit pénal européen qui exige d'être discuté, c'est celui de la langue. Dans un système communautaire de droit pénal européen, une langue véhiculaire me semble nécessaire. Parce qu'une langue est en même temps un langage juridique, différentes langues nous mènent à de différentes interprétations. L'utilisation des termes comme opzet et nalaten en néerlandais, ont pour suite automatiques une interprétation juridiquement plus néerlandaise ou, si vous voulez, belge. Tous ceux qui ont de l'expérience en matière de comparaison juridique savent que des termes comme intent et omission nous mènent dans des concepts juridiques totalement différents et des contextes différents. Ces différences n'ont pas de place dans une forme de maintien européenne. L'Union européenne se pose ellemême beaucoup de problèmes en déclarant chaque langues d'un pays membre, une langue authentique. ${ }^{65}$ Cela comprendra à partir de demain 20 langages !

Si une certaine langue est choisie, un langage juridique européen pourra naître. Il est évident que l'anglais est la langue de choix. Mais cela aussi peut poser des problèmes, car je pense que la structure communautaire du droit pénal devrait être plutôt oriente vers un système de civil law. Mais les expériences des cours internationaux nous apprennent que normalement un nouveau système peut naître, trouvant une balance entre les différents systèmes qui en ont était le berceau. L'anglais devrait donc, dans ce contexte, se différencier de son contexte national. Mais avant que tout ceci soit réalisé et alors que l'application du droit pénal est purement national, la langue néerlandaise est la langue juridique et académique. Cela veut donc dire que l'éducation et la recherche pénale doivent prendre place en néerlandais, et pour la même raison (l'original de) ce monologue l'est de même.

\footnotetext{
${ }^{64}$ A.M. van Kalmthout en P.J.P. Tak, Ups en downs van de minimumstraf, Een verkennende studie naar het voorkomen van minimumstraffen in Frankrijk, België, Duitsland, Engeland en Wales, Nijmegen 2003.

${ }^{65}$ W.C. van Binsbergen, Integratie, Een revolutie? Discours inaugurateur Utrecht 1963, p.12.
} 


\section{L'européanisation de la protection juridique}

Dans mon exposé sur les limites de juridiction et sur le déploiement d'une compétence pénal européenne, je n’ai pas encore mentionné les protections des droits de l'homme qui en sont inséparable. L'union européenne oublie parfois encore qu'un maintien juridique transnational exige une protection juridique différente. Tout d'abord les situations dans lesquelles les Etats coopèrent pénalement. La protection des droits de l'homme et encore très nationale. Un changement pour s'adapter à la nouvelle situation est très important. ${ }^{66}$ Orie s'en est déjà aperçu en 1983 et l'appelle, dans son fameux écrit, "La brèche systématique » de la protection des droits de l'homme dans la coopération internationale pénale. ${ }^{67}$ Dans le droit de coopération internationale en matière pénale, le suspect et les autres personnes involvées ne sont pas protégées dans aucun des deux Etats. Dans l'Etat exigeant le suspect, il pourra difficilement se plaindre d'injustice dans un autre Etat. Dans l'Etat requeré, il manque souvent une protection de ce droit car la procédure n'a souvent pas encore commencé. Le refus d'accepter l'existence d'un maintien transnational crée une brèche dans le système de protection des droits. Nous pouvons donc dire que de ce point de vue, un maintien pénal purement national ne peut que défavoriser la protection des droits d'un suspect. Et deux systèmes parallèles de protection juridique limite la vraie protection. Un plus un serait donc zéro.

La reconnaissance mutuelle des actes d'Etat devrait couvrir chaque aspect de la coopération sans exception de responsabilité envers la Convention européenne des Droits de l'Homme. L'Etat utilisant les services d'un autre devrait être responsable pour les actes de cet autre Etat. Les Etats coopérant devront donc porter la responsabilité des uns et des autres. L'aspect arbitraire de la situation actuelle devrait disparaître avec ça. Pour le moment, un suspect s'apercevant qu'une enquête est menée sur lui, ne peut souvent pas savoir envers quel Etat il peut diriger ces plaintes éventuelles. La Cour européenne des Droits de l'Homme et la (anciene) Commission Européene de Droits de l'Homme ont tous les deux refusés de reconnaître une responsabilité commune de plusieurs Etats. ${ }^{68}$

Un exemple illustrant est le cas Sari. La cour européenne des droits de l'homme a refusé une plainte dirigée envers le Danemark et la Turquie. La plainte se porte sur une violation de durée de procédure acceptable. La procédure avait duré plus de 10 ans mais la responsabilité pour cette période fus partager entre les deux pays par la cour, et les deux périodes isolés ne violaient pas l'exigence de durée raisonnable. ${ }^{69} \mathrm{La}$ tache du plaintif ne devrait pas être la détermination de l'Etat étant responsable pour la violation des droits de l'homme. Finalement ce qui compte est la violation par les deux Etats par leurs actions conjointe. Selon article 1 de La Convention européenne des Droits de l'Homme, les Etats sont aussi conjointement responsable pour la prévention de violations des droits de l'homme. Je vois ceci comme une

\footnotetext{
66 Sur la position de défense dépassant les frontières: Spronken, A place of greater safety, Bespiegelingen over een Europees Statuut voor de strafrechtsadvocaat, discours inaugurateur Maastricht 2003.

${ }^{67}$ A.M.M. Orie, De verdachte tussen wal en schip of de systeem-breuk in de kleine rechtshulp, in: Bij deze stand van zaken, bundel opstellen aangeboden aan A.L. Melai, Arnhem 1983, p.351-361.

${ }^{68}$ A.H. Klip, Die EMRK und die internationale Zusammenarbeit in Strafsachen, Universität Halle, sous presse. A.H. Klip, The Decrease of Protection under Human Rights Treaties in International Criminal Law, 68 Revue Internationale de Droit Pénal 1997, p.291-310.

${ }^{69}$ Cour européenne des Droits de l'Homme, 8 novembre 2001, Sari contre le Denemark et le Turquie, 21889/93, par.91 et 99 .
} 
responsabilité entièrement portée par tous les intéressés. Avant la décision pénale un suspect devrait avoir des moyens juridiques dans tous les Etats concernés.

La ou l'union européenne maintiendra elle-même l'ordre et poursuivra judiciairement, il est absolument nécessaire que l'union adhère á la Convention Européenne des Droits de l 'Homme. Cela offrera des droits come dans les traditions juridiques des états membre et beaucoup plus protection que la selection des droits de l'homme fait à une Chartre des droits fondamentaux de l'Union Européene et au projet de constitution de l'union européenne. ${ }^{70}$

\section{En conclusion}

Je vais en conclure avec ceci. Encore beaucoup d'eau devra couler dans La Meuse avant que l'union européenne en arrive à une formation plus systématique du droit pénale. Ceci a tout a faire avec l'aspect institutionnel de la réalisation d’un droit pénal européen et avec la question de la possibilité politique. Le soi-disant débâcle de décembre dernier avant la création de la constitution européenne ne pourrais être que favorable pour le développement juridique. Le législateur européen a certainement besoin d'un peu plus de temps pour perfectionner ces instruments juridiques. La principale exigence d'un système est le respect de droits fondamentaux et un droit pénal maintienable, ce droit pénal peut ce portait un peu moins bien, alors seulement il pourra jouer un rôle important dans la société européenne et néerlandaise.

\footnotetext{
${ }^{70}$ Articles II-47 - 50 projet de constitution de l'Union européenne et articles 47-50 Chartre des droits fondamentaux de l'Union européenne.
} 